\title{
An Efficient Adaptive Cell Sectoring Technique for Non-Uniform Traffic in DS-CDMA Systems
}

\author{
JIHUI ZHANG and BO LI* \\ Department of Computer Science, The Hong Kong University of Science and Technology, Clear Water Bay, Kowloon, Hong Kong \\ JIANGCHUAN LIU \\ School of Computing Science, Simon Fraser University, Burnaby, British Columbia Canada
}

Published online: 30 December 2005

\begin{abstract}
Cell sectorization has been shown as a promising technique to improve the overall capacity in direct sequence code division multiple access (DS-CDMA) systems. It has been further demonstrated that the use of adaptive antenna arrays with dynamic cell sectoring is particularly suitable for non-uniformly distributed users. In this paper, we first re-formulate cell sectoring into an optimization problem and solve it with dynamic programming algorithm. We next show that this has two major practical drawbacks: the complexity and oscillation of users between neighboring sectors. We then present an efficient Cluster-based Sectoring (CS) algorithm for adaptive cell sectorization to overcome these two inefficiencies: Firstly, the computation complexity of CS algorithm is much lower than that of the optimal sectoring algorithm. In particular under high-density case, the complexity is bounded and does not depend on the number of users in a cell; Secondly, the CS algorithm maintains the excellent property of avoiding sector boundaries frequently crossing those users closely located within short angular distances. In addition, we also investigate the support for multi-rate applications with the proposed CS algorithm. Through extensive experimental study, we find that the performance of proposed CS scheme obtains comparable performance with greatly reduced complexity when comparing to the optimal solution.
\end{abstract}

Keywords: adaptive sectorization, CDMA, power control, interference management

\section{Introduction}

Direct sequence code division multiple access (DS-CDMA) technique has emerged as one of the leading standards for the air interface of the third-generation cellular networks [3]. In DS-CDMA systems, all users share the universal frequency band and the channel capacity is interference-limited. Cell sectoring has been shown as a promising technique to alleviate the co-channel interference and enhance system capacity [8]. It uses antenna arrays at the base station to separate the users into several sectors, where each sector is served by one directional antenna array. Since only signals from a subset of the users are received at each antenna array, the interference among different users might be greatly reduced compared to a single omni-directional antenna system [12].

Traditional sectoring approaches divide the cell into equal width sectors, which is ideal for uniform traffic load [11]. However, for non-uniform traffic, such fixed sectoring approach often fails to obtain the anticipated capacity gain, and the sectors with high-density traffic may suffer high outage probability. This deficiency can be eliminated by the use of adaptive cell sectorization. In an adaptive sectorization, the

\footnotetext{
* Corresponding author.

E-mail: bli@cs.ust.hk
}

sector boundaries can be adaptively adjusted according to the geographic distribution of the call traffic. This is particularly well justified for the recent developments on fixed wireless systems, such as Wireless Local Loops (WLL), in which users locations, and thus their uplink gains to the based station, vary slowly compared to the high mobility networks. In such a case, it is possible to optimally sector the cells based on the traffic information, and adjust the antenna boundaries when the traffic pattern changes.

Generally speaking, lower transmission power from users in one cell results in less interference to neighboring cells. Thus, the capacity for the whole system will be improved. In the adaptive cell sectorization, the fundamental problem is to determine the direction and width of each sector in order to minimize the Total Transmission Power (TTP), while at the same time, maintaining each user's QoS requirement in terms of a lower bound Signal-to-Interference Ratio (SIR) experienced by users in DS-CDMA systems. We refer this problem as the Optimal Sectoring (OS) problem.

As the capacity of a DS-CDMA cellular network is reverse-link limited [4], in this paper, we mainly focus on the cell sectoring on the uplink in DS-CDMA cellular networks. We first formulate cell sectoring into an optimization problem and solve it with dynamic programming algorithm. With the information on the traffic distribution and number of 
sectors, we can optimally design the sector boundaries such that minimum TTP can be achieved in the systems subject to the SIR constraint for each user.

The above solution is optimal; however, it practically incurs high computation complexity. Hence, it does not scale well to high-density cells. We believe that, for such an adaptation system, reducing the execution time of the sectoring algorithm is also of primary importance. Furthermore, we observe that, in practice, the sector boundaries should better be across low-density regions. The reasons are as follows: Firstly, separating very close users is impractical given the difficulty in achieving precise directivity; Secondly, if a boundary goes through an extremely high-density region, very frequent oscillations of users across the boundary might potentially occur, resulting in non-stable sector partition; in other words, the adaptive sectoring algorithm needs to be executed very frequently. With these considerations, we then propose a Cluster-based Sectoring (CS) algorithm to reduce the computation complexity under high-density traffic distribution, while at the same time trying to avoid the sector boundaries across those neighboring users whose physical locations are close in the angular distances. The dynamic programming technique is also applied in the CS algorithm to solve the MinTTP problem.

The basic idea in the proposed CS algorithm is to partition the users into several clusters, where the users in the same cluster are geographically nearby in the angular distance with their neighboring users. For a high-density cluster that exceeds some threshold, we apply an Angular-based Partitioning (AP) algorithm to further separate it into several sub-clusters to reduce the TTP. Generally speaking, the number of clusters is much smaller than the number of users, but larger than that of sectors. We then apply a dynamic programming (DP) algorithm on the clusters, not the users, to find the sector partition. The CS algorithm is not globally optimal, but its time complexity is significantly reduced compared with its optimal counterpart. In particular, under the high-density traffic distribution, the main complexity from DP calculation does not depend on the number of users in the cell, making it practically feasible. We carry out detailed analysis of the complexity for the algorithm, and with extensive simulations, we find that, compared to the optimal solution, the complexity of CS algorithm is greatly reduced. The performance degradation of the sub-optimal solution is limited and usually less than $10 \%$, which is particularly efficient from a practical point of view.

The rest of this paper is organized as follows. Section 2 introduces related work. Section 3 describes the system model and formulates the optimal sectoring problem. In Section 4, we use a dynamic programming algorithm to solve the MinTTP optimization. The cluster-based sectoring algorithm and detailed complexity analysis are presented in Section 5. In Section 6, we apply the algorithm to multi-rate transmission in MC-CDMA system. In Section 7, we discuss some practical issues related to cell sectoring. We investigate the performance of our algorithm through numerical simulations in Section 8. Finally, we conclude the paper in Section 7.

\section{Related work}

It has been shown in [2,11] that the use of antenna arrays at base-station greatly increases the system capacity by reducing the co-channel interference. However, simple fixed sectorization that splits a cell into sectors with equal width is not adaptive to the highly non-uniform traffic load situations, namely "Hot Spot".

With this observation, increased amount of works have been focusing on the adaptive cell sectorization to achieve the improved capacity gain with the existence of "Hot Spots" in the system. In [5], the sectors are rotated and resized adaptively to equalize the uplink carrier-to-interference ratio or the total downlink transmitted power in each sector, and it shows that the performance is improved under non-uniformly distributed traffic scenario. In [1], network architecture for CDMA cellular systems based on adaptive sector coverage has been proposed. The sector angle is changed according to the traffic intensity in a sector to avoid excessive blocking due to temporary non-uniformity of traffic across the cell. The paper also employs call admission control to obtain better performance in maintaining the QoS in overloaded sectors. In [15], the authors present a dynamic tilted-antenna mechanism, which tilts the antennas of the hot spot and its adjacent cell sectors, for sectored cells in CDMA cellular networks to mitigate the congestion in a hot-spot sector, thus improving the system capacity.

In [13], the paper presents an optimized cell sectorization scheme to maximize the system capacity, in which the optimal sectoring (OS) problem was transformed into the shortest path problem and then solved by the well-known Dijkstra's algorithm. In our scheme, we adopt the similar formulation, and use a dynamic programming technique to solve the OS problem. Our solution is much more straightforward without the overhead of graphic mapping. Furthermore, our main focus in this paper is to obtain better adaptation and less complexity. We will discuss the tradeoff between the computation complexity and performance in the later sections.

\section{System model and formulation}

\subsection{System model}

In this paper, we mainly focus on sectoring in the uplink of a single cell DS-CDMA system, where each user specifies a minimum tolerable QoS which can be mapped into a lower bound Signal-to-Interference Ratio (SIR) level. Adaptive antenna arrays are applied at the base station to partition the cell into multiple sectors, where each directional antenna receives signals from the users within the particular sector it serves, resulting in a spatial isolation of users in the cell. Assume ideal directivity for the adaptive antenna arrays, and thus no interference occurs between sectors. As a result, each user's receiver will only experience interference from other users 
Table 1.

List of notations.

\begin{tabular}{|c|c|}
\hline$N$ & Total number of sectors \\
\hline$K$ & Total number of users in the cell \\
\hline$\eta$ & Thermal noise power \\
\hline$\alpha_{j}$ & Speech activity factor of the user $j$ \\
\hline$G_{i}$ & $\begin{array}{l}\text { Processing gain of the user } i . G_{i}=W / R_{i} \text { where } W \text { is the } \\
\text { total bandwidth and } R_{i} \text { is the bit rate }\end{array}$ \\
\hline$\chi_{i}$ & Set of users in $i$-th sector \\
\hline$\chi(i)$ & Set of users in the same sector with user $i$ \\
\hline$\gamma_{i}$ & Minimum SIR requirement of user $i$ \\
\hline$p_{i} h_{i}$ & $\begin{array}{l}\text { Received power by base station transceiver from user } i \text {, } \\
\text { where, } p_{i} \text { is the transmitted power and } h_{i} \text { is the channel } \\
\text { gain for the user } i\end{array}$ \\
\hline$K_{i}$ & Number of users in $i$-th sector \\
\hline$K_{C_{t}}$ & Number of users in the cluster $C_{t}$ \\
\hline$M_{i}$ & Number of clusters in sector $i$ \\
\hline$\chi_{i}^{C}$ & Set of clusters in sector i \\
\hline$\chi^{C}(i)$ & Set of clusters in the same sector with cluster $i$ \\
\hline$\delta$ & Angle distance for Cluster-based Sectoring Algorithm \\
\hline$\tau$ & $\begin{array}{l}\text { Angular changing interval in a cluster for Angular-based } \\
\text { Partitioning Algorithm }\end{array}$ \\
\hline$\kappa$ & $\begin{array}{l}\text { Set of splitting points in Angular-based Partitioning } \\
\text { algorithm for a cluster }\end{array}$ \\
\hline
\end{tabular}

within the same sector. Our objective here is to find the best partition of the cell in order to minimize the total transmission power (TTP) for the whole system, while at the same time ensuring SIR requirement for each user. We first list the notations used in this paper in Table 1 before formally describing MinTTP problem.

\subsection{Optimal sectoring problem}

Let $K$ be the number of users and $N$ the number of sectors partitioning the cell. The SIR requirements for user $i$ at the base station receiver can be represented by the bit energy to total interference plus thermal noise density ratio, $E_{b} / N_{0}[1]$

$$
\left(\frac{E_{b}}{N_{0}}\right)_{i}=\frac{G_{i} p_{i} h_{i}}{\sum_{j=1, j \in \chi(i), j \neq i}^{K} \alpha_{j} p_{j} h_{j}+\eta} \geq \gamma_{i} .
$$

Assume perfect power control, thus the received power at the base station from each user should be the same. A common target SIR requirement $\gamma$ is assigned to all the users in the cell, i.e. $\gamma_{i}=\gamma$. Further assume identical voice activity factor $\alpha_{i}=\alpha$ and bit rate $R_{i}=R$. As $G_{i}=W / R_{i}$, this results in the same processing gain $G_{i}=G$. The optimization problem can be formulated as follows:

$$
\varphi_{S P}(k, n)= \begin{cases}\frac{\eta \gamma}{G-(k-1) \alpha \gamma} \sum \frac{1}{h_{j}}, & n=1,1 \leq k<\frac{G}{\alpha \gamma}+1 \\ \min _{n-1<i \leq k}\left\{\varphi(i-1, n-1)+\frac{\eta \gamma}{G-(k-i) \alpha \gamma} \sum_{j \in \chi(i)} \frac{1}{h_{j}}\right\}, & n>1, n \leq k<\frac{G}{\alpha \gamma}+i \\ +\infty, & \text { Otherwise }\end{cases}
$$

$$
\begin{array}{cl}
\min _{\theta, P} \sum_{s=1}^{N} \sum_{i \in \chi_{s}} p_{i}, \\
\text { s.t. } \quad \frac{G p_{i} h_{i}}{\sum_{j=1, j \in \chi(i), j \neq i}^{K} \alpha p_{j} h_{j}+\eta} \geq \gamma, \quad i=1, \ldots K, \\
\\
P \geq 0, \quad P=\left(p_{1}, \ldots p_{K}\right), \\
\sum_{i=1}^{N} \theta_{i}=2 \pi, \quad \theta=\left(\theta_{1}, \ldots \theta_{N}\right)
\end{array}
$$

It has been shown that the system capacity is maximized when each mobile's transmitted power level is controlled so that its signal arrives at the base station with the minimum required SIR. In addition, within each sector, the received power for each user is controlled to be equal at the base station, i.e., $p_{i} h_{i}=p_{j} h_{j}$, where $j \in \chi(i)$ [12]. Therefore, we can rewrite Formulation (2) as follows:

$$
\begin{aligned}
\text { (MinTTP) } \min _{\theta, P} & \sum_{i=1}^{N} \frac{\eta \gamma}{G-\left(K_{i}-1\right) \alpha \gamma} \sum_{j \in \chi_{i}} \frac{1}{h_{j}}, \\
\text { s.t. } \quad & K_{i}<G / \alpha \gamma+1, \quad \sum_{i=1}^{N} K_{i}=K, \\
& \sum_{i=1}^{N} \theta_{i}=2 \pi \quad \theta=\left(\theta_{1}, \ldots \theta_{N}\right) .
\end{aligned}
$$

\section{Optimal solution}

In this section, we present a dynamic programming (DP) algorithm to solve the above optimization problem. The DP algorithm is also the basis for the cluster-based sectoring in subsequent sections.

As discussed in Section 3, with the information on the $K$ user distribution and predefined sector number $N$ in the cell, the optimization problem can be represented as:

$$
\min _{\theta, P} \sum_{i=1}^{N} \sum_{j \in \chi_{i}} p_{j}=\min _{\theta, P} \sum_{i=1}^{N} \frac{\eta \gamma}{G-\left(K_{i}-1\right) \alpha \gamma} \sum_{j \in \chi_{i}} \frac{1}{h_{j}}
$$

Without loss of generality, we can choose one user as the start user $U_{1}$. From this user, label counter-clockwise the remaining users from $U_{2}$ to $U_{k}$, where $k$ is the total number of users. We set the start-partitioning point between user $U_{k}$ and user $U_{1}$, denoted as $S P=\left(U_{k}, U_{1}\right)$. Let $\varphi_{S P}(k, n)$ represent the MinTTP of $k$ users partitioned with $n$ sectors for the given start partitioning point $S P$. We have the following recurrence relation, 
Where, $i$ is the partition point for the $n$-th sector (including $\left.U_{i}\right)$ and $\chi(i)$ is the set of consecutive users in the same sector with user $i$, i.e. $\left\{U_{i} U_{i+1} \ldots U k\right\}$. Please refer to Appendix A for the correctness proof of the recurrence function (5).

Lemma 1. $\min _{1 \leq s \leq K} \varphi_{S P}(K, N)$ is the solution to the optimization problem MinTTP (3), where the start partitioning point

$$
S P= \begin{cases}\left(U_{K}, U_{1}\right) & s=1 \\ \left(U_{s-1}, U_{s}\right) & s>1 .\end{cases}
$$

The proof of the lemma is obvious by changing the start partitioning boundary continuously, one for each user, and computing the similar optimal solution $\varphi_{S P}(k, n)$ according to the $k$ consecutive start partitioning point in a cyclic way. For the system with $K$ users to be partitioned into $N$ sectors, $\operatorname{MinTTP}=\min _{1 \leq s \leq K} \varphi(K, N)$.

The complexity of dynamic programming algorithm on computing each $\varphi(K, N)$ is $\mathrm{O}\left(N K^{2}\right)$, so the complexity on computing the optimal solution for MinTTP is $\mathrm{O}\left(N K^{3}\right)$, which is the same as that of the result obtained in [13].

\section{The cluster-based sectoring algorithm}

In this section, we propose a Cluster-based Sectoring (CS) algorithm as a sub-optimal solution to the MinTTP problem (3), which might greatly reduce the complexity in the optimal solution and guide the sector boundaries across the low-density regions. We first divide the users into several clusters based on a nearby criterion, in terms of the relative angular distances between neighboring users. Here, we assume that each sector contains at least one user, and the users within a particular cluster are placed into the same sector. In addition, given the MinTTP objective and the capacity constraint $K_{i}<G / \alpha \gamma+$ 1 , we will partition those "congested" clusters, i.e. clusters

$\varphi_{S P}^{c}(m, n)= \begin{cases}\frac{\eta \gamma}{G-\left(\sum_{t=1}^{m} K_{C_{t}}-1\right) \alpha \gamma} \sum_{j=1}^{m} h_{c_{j}}, & \text { Appendix B. } \\ \min _{n-1<i \leq m}\left\{\varphi^{c}(i-1, n-1)+\frac{\eta \gamma}{G-\left(\sum_{t=i}^{m} K_{C_{t}}-1\right) \alpha \gamma} \sum_{j=i}^{m} \frac{1}{h_{C_{j}}}\right\}, & n>1,1 \leq \sum_{t=i}^{m} K_{C_{t}}<\frac{G}{\alpha \gamma}+1 \\ +\infty, & \text { Otherwise }\end{cases}$ containing too many users, into some sub-clusters with an Angular-based Partitioning algorithm and put the sub-clusters back into the overall cluster set. A dynamic programming algorithm is then applied on the whole set of clusters to obtain the MinTTP partition.

\subsection{Cluster-based sectoring}

A cluster is a sequence of users, where each user's angular distance to its neighbor(s) is less than a predefined threshold $\delta$. For all the users in the cell, we first divide them into a set of clusters. This can be done in two steps, namely coarse clustering and angular-based partitioning. The coarse clustering algorithm is performed as follows: Firstly, arbitrary choose one user as the starting user for the first cluster. From this user, we check the remaining users in the cell one by one in a counter-clockwise manner. If the current user's angular distance to the previous one is less than $\delta$, it will be added into the current cluster, i.e. the same cluster the previous user stays. Otherwise, we start a new cluster by setting the current user as the first user and repeat the above step for user addition. This procedure will stop when each user in the cell has been included into one and only one cluster. Finally, if the angular distance between the starting user of the first cluster and the end user of the last cluster is less than $\delta$, we combine these two clusters into one cluster. After the coarse clustering, the clusters containing too many users will be further partitioned by an angular-based partitioning algorithm, with details given in the next subsection.

When both coarse clustering and angular-based partitioning are done, we would obtain a set of clusters. Sectoring on the clusters, namely cluster-based sectoring, is then performed for MinTTP problem, which can be shown as follows:

$$
\min _{\theta, P} \sum_{i=1}^{N} \frac{\eta \gamma}{G-\left(\sum_{C_{t} \in \chi_{i}^{C}} K_{C_{t}}-1\right) \alpha \gamma} \sum_{C_{j} \in \chi_{i}^{C}} \frac{1}{h_{C_{j}}}
$$

where $K_{C_{t}}$ is the number of users in the cluster $C_{t}, \chi_{i}^{C}$ is the set of clusters in the sector $i$ and $\frac{1}{h_{C_{j}}}=\sum_{k \in C_{j}} \frac{1}{h_{k}}$.

A dynamic programming algorithm similar to what we use in the optimal sectoring (Section 4) is then designed with the recurrence relation shown as (7), by regarding a cluster as one particular integrated user. Correctness proof can be found in Appendix B.

Where, $m$ is the size of the clusters, $C_{i}$ is the partition point for the $n$-th sector (including $C_{i}$ ) and $\chi^{C}(i)$ is set of clusters in the same sector with cluster $C_{i}$. Assume a total of $M$ clusters to be partitioned into $N$ sectors in the system, $\min _{1 \leq s \leq M} \varphi_{S P}^{c}(M, N)$ is the solution to the optimization problem (6), where the start partition point

$$
S P=\left\{\begin{array}{cc}
\left(C_{M}, C_{1}\right) & s=1 \\
\left(C_{s-1}, C_{s}\right) & s>1
\end{array} .\right.
$$




\subsection{Angular-based partitioning}

When some high-density regions or "hot spots" exist in the cell, the coarse clustering method might result in very large clusters. If too many users are clogged into one cluster, thus placed into the same sector later, they would likely incur great interfere among one another, resulting in high TTP. In certain situation, the number of users in one cluster may exceed the capacity constraint $\frac{G}{\alpha \gamma}+1$. It is thus necessary to further partitioning those large clusters into some smaller sub-clusters.

For a large cluster that needs partitioning, we first roughly decide the number of users supposed in a sector with equalized sectoring [13]. The number of sub-clusters is determined by the heuristic $m_{C_{i}}=\left\lfloor\frac{K_{C_{i}}}{K / N}+\sigma\right\rfloor$, where $\sigma$ is a predefined threshold for adjustment. Based on user distribution information and number of sectors, we further split the cluster by an angular-based partitioning algorithm with the objective to minimize the total transmission power of all users in the original cluster. Note that, due to the physical constraint of directivity accuracy, it does not make too much practical sense to set two neighboring boundaries within a precision threshold. So, we only allow the sectoring boundaries to take discrete incremental angular values. In our algorithm specifically, we assume the minimum angular interval between any pair of sectoring boundaries, i.e. the precision threshold, to be $\tau$, namely the Angular Changing Interval. Assume $\kappa$ is the set of all the feasible partitioning intervals (i.e. at least one user in each interval). Our algorithm is then applied to set $\kappa$, instead of the set of users in the cluster, to split the original cluster into $m_{C_{i}}$ sub-clusters. This can also be done by a similar dynamic programming algorithm. Note here, instead of partitioning based on the users, we set the partition on the feasible splitting intervals represented by $\kappa$. The recurrence relation $\varphi^{A}(l, n)$ is written as follows:

$$
\varphi^{A}(l, n)= \begin{cases}\frac{\eta \gamma}{G-\left(K^{C}-1\right) \alpha \gamma} \sum_{j=1}^{K^{C}} \frac{1}{h_{j}}, & n=1,1 \leq K^{C}<\frac{G}{\alpha \gamma}+1 \\ \min _{n-1<i \leq l}\left\{\varphi^{A}(i-1, n-1)+\frac{\eta \gamma}{G-\left(K_{n}-1\right) \alpha \gamma} \sum_{j \in \chi_{n}} \frac{1}{h_{j}}\right\}, & n>1,1 \leq K_{n}<\frac{G}{\alpha \gamma}+1 \\ +\infty, & \text { Otherwise }\end{cases}
$$

where, $n$ is the number of sectors; $l$ is the size of splitting set $\kappa ; K^{C}$ is the number of users in the original cluster; $K_{n}$ is the number of users in sector $n$, i.e. number of users from splitting point $i$ to $l$. Later on, the sub-clusters are placed back into the overall cluster set for the cluster-based sectoring, as discussed in the previous subsection.

\subsection{Complexity analysis}

In Section 4, it has been shown that the complexity of the optimal sectoring algorithm is $\mathrm{O}\left(N K^{3}\right)$. In this subsection we will show that the complexity for the cluster-based sectoring algorithm is much lower. The computation of our algorithm can be divided into two parts:

\subsubsection{Coarse clustering and angular-based partitioning}

The coarse clustering only involves the operation of user scanning, thus the complexity of it is simply $O(K)$. When performing angular-based partitioning on the very large clusters, as the algorithm is applied to a cluster with sequenced users, the starting partitioning point is fixed to the first user of the cluster. Let $M^{\prime}$ be the number of clusters requiring further partition, $K_{C_{i}}$ be the number of users in cluster $C_{i}$, and $m_{C_{i}}$ be the number of sub-clusters for $C_{i}$. In this paper, we set $m_{C_{i}}=\left\lfloor\frac{K_{C_{i}}}{K / N}+\sigma\right\rfloor$, where $\sigma$ is a predefined threshold for adjustment. Let $A_{C_{i}}$ be the overall angular distance between the start and end user in $C_{i}$. As at least one user in each sector, we do not consider the angular splitting intervals without any user in between. Thus the size of $\kappa$ is $\min \left\{K_{C_{i}}, A_{C_{i}} / \tau\right\}$, and the complexity for this part is given by $\sum_{i=1}^{M^{\prime}} O\left(m_{C_{i}} \cdot\left(\min \left\{K_{C_{i}}, A_{C_{i}} / \tau\right\}\right)^{2}\right)$, subject to $M^{\prime} \leq M, \sum_{i=1}^{M^{\prime}} A_{C_{i}} \leq 2 \pi$, and $\sum_{i=1}^{M^{\prime}} K_{C_{i}} \leq K$. For the extreme case where one large cluster includes all the users, we have $\sum_{i=1}^{M^{\prime}} O\left(m_{C_{i}} \cdot \min \left\{K_{C_{i}}, A_{C_{i}} / \tau\right\}^{2}\right) \leq O(N$. $\left.\min \{K, 2 \pi / \tau\}^{2}\right) \leq O\left(N K^{2}\right)$.

\subsubsection{Dynamic programming on a set of clusters}

As the dynamic programming algorithm is applied directly to the clusters, the complexity can be largely reduced in the non-uniform high-density case where many users gather together closely. Let $M(M<K)$ be the number of clusters in the cell, the complexity to compute $\varphi_{S P}^{c}(M, N)$ is $O\left(N M^{2}\right)$, thus the MinTTP solution (i.e. $\min _{1 \leq s \leq M} \varphi_{S P}^{c}(M, N)$ ) could be obtained in $O\left(N M^{3}\right)$. Here, $M$ is bounded by $\{2 \pi / \delta, K\}$.

Combing these two parts, the total complexity of the algorithm is $K+\sum_{i=1}^{M^{\prime}} O\left(m_{C_{i}} \cdot \min \left\{K_{C_{i}}, A_{C_{i}} / \tau\right\}^{2}\right)+O\left(N M^{3}\right)$. It is obvious that the computation effort for optimal solution

mainly comes from the cube of user number, i.e. $K^{3}$. Generally speaking, the complexity of the coarse clustering and angular-based partitioning (first part) can be ignored as it is one order of magnitude less than that of the cluster sectoring part. Furthermore, in the extremely high-density case, the complexity can be bounded for specified $\delta$ and $\tau$, and does not depend on the number of users. This greatly decreases the computation complexity. On the other hand, in a sparse user distribution, our algorithm gives the optimal solution, and its complexity is no higher than the user-based optimal sectoring algorithm. 


\section{High rate data services}

Next generation wireless cellular networks are expected to accommodate multimedia services. To provide multi-class service transmitting at variable rate in DS-CDMA systems, two main techniques are widely deployed, namely Variable Spreading Factor (VSF) and Multi-Code (MC). In a VSFCDMA system, higher transmission rate can be obtained by lowering processing gain, thus requiring greater transmission power to ensure SIR constraint. In a MC-CDMA system, users that need higher data rates make use of multiple codes, each with a basic bit rate, for transmission in parallel. In this section, we focus on the multi-rate transmission with MC scheme and show that the previously mentioned algorithm can be easily adjusted for multiple rate transmission. Furthermore the result can also be readily extended for VSF-CDMA systems.

As all signals over the radio interface are transmitted at a basic rate, namely $R_{b}$ with one chip code, the spread processing gain over each code channel is a constant. Assume each user associates with $c_{i}$ codes for transmission at rate $c_{i} b_{i}$ and the same power $p_{i}$ is allocated to each of the $c_{i}$ code words. Further assume that orthogonal codes are assigned for each user's transmission such that for one particular code channel, no self-interference experienced from the other $\left(c_{i}-1\right)$ parallel code channel in use. This can be done by subcode catenation technique [7, 10]. Again, assume identical voice activity factor $\alpha_{i}=\alpha$ and the same SIR requirement $\gamma_{i}=\gamma$ for each user. The optimal formulation can be written as:

$$
\begin{array}{ll}
\min _{\theta, P} \sum_{s=1}^{N} \sum_{i \in \chi_{s}} c_{i} p_{i}, \\
\text { s.t. } & \frac{p_{i} h_{i} / R_{b}}{\left(\sum_{j=1, j \in \chi(i), j \neq i}^{K} \alpha c_{j} p_{j} h_{j}+\eta\right) / W} \\
& P=0, \quad i=1, \ldots K, \\
& P=\left(p_{1}, \ldots p_{K}\right), \\
& \sum_{i=1}^{N} \theta_{i}=2 \pi, \quad \theta=\left(\theta_{1}, \ldots \theta_{N}\right) .
\end{array}
$$

Let $G=W / R_{b}$ and assume the same received power for each code in the cell, i.e. $p_{i} h_{i}=p_{j} h_{j}$, then,

$$
\begin{aligned}
\operatorname{MinTTP}: & \min _{\theta, P} \quad \sum_{i=1}^{N} \sum_{j \in \chi_{i}} \frac{\gamma \eta}{G-\gamma \cdot \alpha \cdot \sum_{k \in \chi_{i}, k \neq j} c_{k}} \frac{c_{j}}{h_{j}} \\
\text { s.t. } & \sum_{k \in \chi_{i}, k \neq j} c_{k} \leq \frac{G}{\gamma \cdot \alpha}, j \in \chi_{i}
\end{aligned}
$$

Accordingly, the recurrence relation for the optimal DP solution can be expressed as:

$$
\varphi(k, n)=\left\{\begin{array}{c}
\eta \gamma \sum_{j=1}^{k} \frac{1}{G-\alpha \gamma \sum_{t \neq j} c_{t}} \frac{c_{j}}{h_{j}}, \\
n=1, \sum_{k \neq j} c_{k}<\frac{G}{\alpha \gamma} \\
\min _{n-1<i \leq k}-1<i \leq k\{\varphi(i-1, n-1) \\
\left.+\sum_{j \in \chi(i)} \frac{\eta \gamma}{G-\alpha \gamma \sum_{t \in \chi(i), t \neq j} c_{t}} \frac{1}{h_{j}}\right\}, \\
1<n, \sum_{t \in \chi(i), t \neq j} c_{t}<\frac{G}{\alpha \gamma} \\
+\infty, \quad \text { Otherwise }
\end{array}\right.
$$

Generally speaking, there are finite user classes $\Delta t\left(C_{\max }\right.$ $\geq t \geq 1, C_{\max }$ is the max number of classes the system supported), transmitting at different rates with number of $\operatorname{codes} C^{\Delta_{t}}$, the recurrence relation could be further simplified as,

$$
=\left\{\begin{array}{c}
\eta \gamma \sum_{t=1}^{C_{\max }} \frac{1}{G-\alpha \gamma\left(\sum_{t^{\prime}=1}^{C_{\max }} N^{\Delta_{t^{\prime}}} c^{\Delta_{t^{\prime}}}-c^{\Delta_{t}}\right)} \sum_{j \in \Delta_{t}} \frac{c_{j}}{h_{j}}, \\
1=n, \sum_{t^{\prime}=1}^{C_{\max }} N^{\Delta_{t^{\prime}}} c^{\Delta_{t^{\prime}}}-c^{\Delta_{t^{\prime}}}<\frac{G}{\alpha \gamma} \\
\min _{n-1<i \leq k}-1<i \leq k\{\varphi(i-1, n-1) \\
+\eta \gamma \sum_{t=1}^{C_{\max }} \frac{1}{G-\alpha \cdot \gamma \cdot\left(\sum_{t^{\prime}=1}^{C_{\max }} N_{i}^{\Delta_{t^{\prime}}} c^{\Delta_{t^{\prime}}}-c^{\Delta_{t}}\right)} \\
\left.\times \sum_{j \in \Delta_{t}, j \in \chi(i)}^{C_{\max }} \frac{1}{h_{j}}\right\}, \\
1<n, \sum_{t^{\prime}=1}^{\Delta_{t^{\prime}}} c^{\Delta_{t^{\prime}}}-c^{\Delta_{t^{\prime}}}<\frac{G}{\alpha \gamma} \\
+\infty, \quad \text { Otherwise }
\end{array}\right.
$$

where, $N^{\Delta_{t}}$ is the total number of Class-t' users in the cell; $N_{i}^{\Delta_{t \prime}}$ is the number of Class- $t^{\prime}$ users in the sector $i$.

The sub-optimal cluster-based sectoring algorithm which partitions $m$ clusters into $n$ sectors may be applied as follows, 


$$
\begin{aligned}
& \varphi^{c}(m, n) \\
& \int \eta \gamma \sum_{t=1}^{C_{\max }} \frac{1}{G-\alpha \gamma\left(\sum_{t^{\prime}=1}^{C_{\max }} \sum_{r=1}^{m} N_{C_{r}}^{\Delta_{t^{\prime}}} c^{\Delta_{t^{\prime}}}-c^{\Delta_{t}}\right)} \\
& \times \sum_{j=1, j \in \Delta_{t}}^{m} \frac{C_{j}}{h_{C_{j}}^{\Delta_{t}}}, \\
& 1=n, \sum_{t^{\prime}=1}^{C_{\max }} \sum_{r=1}^{m} N_{C_{r}}^{\Delta_{t^{\prime}}} c^{\Delta_{t^{\prime}}}-c^{\Delta_{t}}<\frac{G}{\alpha \gamma}
\end{aligned}
$$

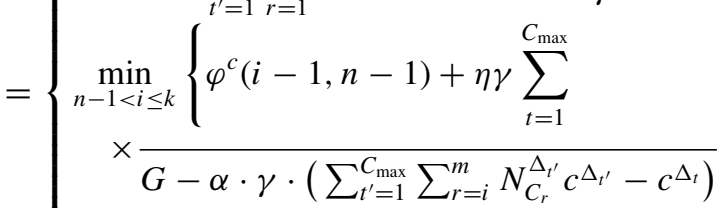

$$
\begin{aligned}
& \left.\times \sum_{j \in \Delta_{t}, j \in \chi(i)} \frac{1}{h_{C_{j}}^{\Delta_{t}}}\right\}, \\
& \begin{array}{c}
1<n, \sum_{t^{\prime}=1}^{C_{\max }} \sum_{r=1}^{m} N_{C_{r}}^{\Delta_{t^{\prime}}} c^{\Delta_{t^{\prime}}}-c^{\Delta_{t}}<\frac{G}{\alpha \gamma} \\
\text { Otherwise }
\end{array}
\end{aligned}
$$

where, $N_{C_{r}}^{\Delta_{t \prime}}$ is the number of Class- $t^{\prime}$ users in cluster $C_{r}$ and $\frac{1}{h_{C_{j}}^{\Delta_{t}}}=\sum_{k \in C_{j}, k \in \Delta_{t}} h_{t}$.

\section{Discussions}

In the previous sections, we consider a single cell DS-CDMA system with perfect power control, cell sectoring, and static radio propagation. To cope with more realistic systems, several practical issues need to be considered.

\subsection{Imperfect sectoring}

In the ideal case, a directional antenna will only receive signals from the users within the sector it covers. In reality, there are always some overlaps for the antenna patterns, incurring cross-interference among neighboring sectors. In other words, the antenna responsible for the current sector may receive signals from those users located in the overlap region of the adjacent sectors. To suppress the additional interference, the transmission power for each user should be increased, resulting in higher MinTTP. This is another driven force to design the sector boundaries across low density regions. To be precise, we could take into account those mobiles in the overlap region as additional interference.

\subsection{Physical constraints}

In practice, we might have to consider transmission power constraint for mobile users, i.e. the power consumption for any user less than a threshold; and spreading angle constraint for antenna patterns, i.e. the sector width within an admissible interval. To ensure the maximum transmission power for each mobile user, one item $p_{i} \leq P_{\max }$ should be considered in the MinTTP formulation. Similarly, $\theta_{\min }<\theta_{i}<\theta_{\max }$ helps ensure the sector width constraint.

\subsection{Imperfect power control and shadowing}

A commonly accepted model for imperfect power control and shadowing is the Log-normal approximation, with power control error $L\left[\sigma_{e}^{2}\right]$ and shadowing effect $L\left[\sigma^{2}\right]$. Here, the variable $10 \cdot \log _{10}\left(L\left[\sigma_{e}^{2}\left(\sigma^{2}\right)\right]\right)$ obey Gaussian distribution of $N\left(0, \sigma_{e}^{2}\left(\sigma^{2}\right)\right)$.

Thus, the SIR requirement can be revised according to some statistical constraint on the outage probability, i.e.,

$$
\begin{aligned}
& \operatorname{Pr}\left\{\frac{L_{i}\left[\sigma_{e}^{2}\right] \cdot p_{i}\left(h_{i} \cdot L_{i}\left[\sigma^{2}\right]\right) / R_{b}}{\left(\sum_{j=1, j \in \chi(i), j \neq i}^{K} \alpha c_{j} p_{j}\left(h_{j} L_{j}\left[\sigma^{2}\right]\right)+\eta\right) / W} \geq \gamma_{i}\right\} \\
& \quad \leq \Gamma_{\text {outage }}
\end{aligned}
$$

where, $\Gamma_{\text {outage }}$ is the acceptable SIR outage probability ensuring transmission quality and $p_{i}\left(h_{i} \cdot L_{i}\left[\sigma^{2}\right]\right)=p_{j}\left(h_{j} L_{j}\left[\sigma^{2}\right]\right)$.

The outage constraint might be approximated by certain distribution to gain transmission power for each user and optimize MinTTP accordingly.

Though the diversity in channel strength has been show to improve the performance by scheduling transmissions with peak channel quality, due to the difficulty in precisely tracking the channel fluctuations, in practice, we have to average out the randomness with decreased performance.

\subsection{Multicell environment}

In previous sections, we consider only the single cell system, ignoring the impact from neighboring cells. To extend our result to the multicell environment, we have to take into account the out-of-cell interference [3]. Assume full knowledge on the user distribution in the interfering cells; we could incorporate some parameters indicating the out-of-cell interference in the SIR formulation and derive the similar dynamic programming algorithms for the MinTTP optimization.

To conclude, the practical concerns listed above may greatly degrade the system performance with lower achievable cell capacity and higher transmission power consumption, and present more challenges on precise prediction and adjustment of the parameters.

\section{Numerical results}

In this section, we investigate the performance of our clusterbased sectoring algorithm through simulations. For comparison, we also implement other two partition algorithms, namely, Optimal Sectoring and Fixed Sectoring. For simplicity, in the later part of the section, we denote OS as the optimal MinTTP result obtained from section II, CS (Clustered Sectoring) as the MinTTP result of cluster-based sectoring algorithm described in Section 3 and FS as the TTP of the 

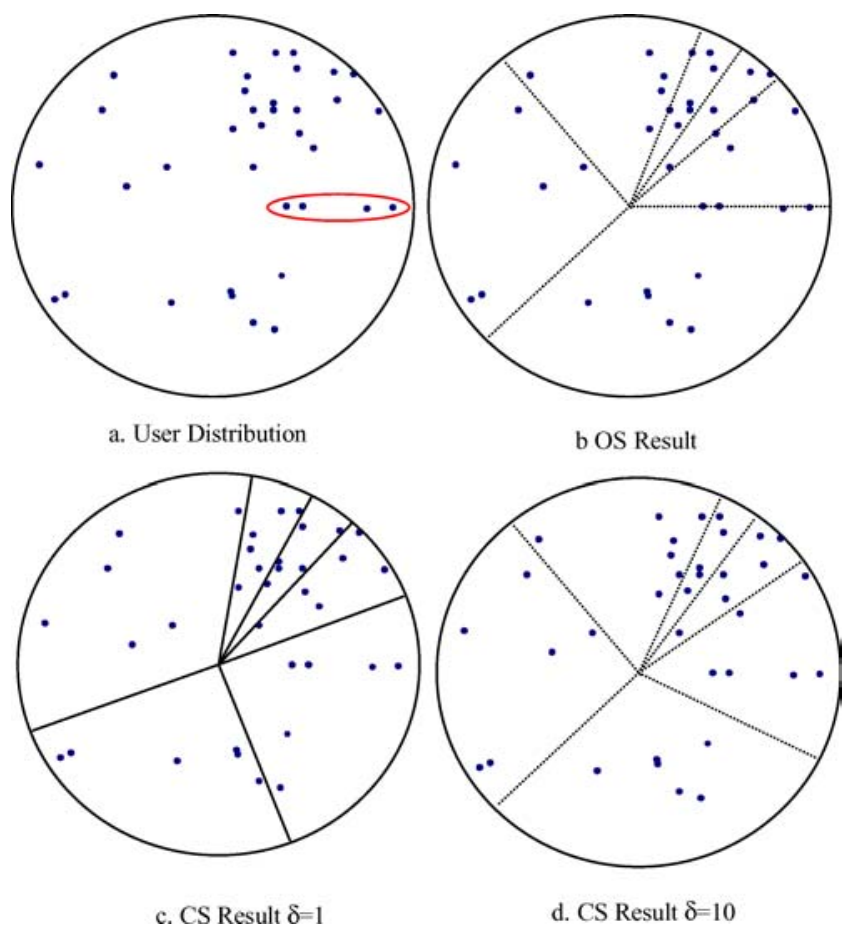

Figure 136 users, 6 sectors. The lines represent the sectoring boundaries. The ellipse shows one of the clusters.

traditional fixed sectoring, where all the sectors in a cell are of the same size and fixed orientation.

In our experiment, we use the following parameter settings: processing gain, $G=64$; SIR requirement $\gamma=7 \mathrm{~dB}$; voice activity $\alpha=0.8$; thermal noise power $\eta=1 \mathrm{e}-12$; angular changing interval in a cluster for the angular-based partition algorithm $\tau=2 \pi / 360$ (reflect the accuracy of antennas). The channel gain $h$ is calculated with a simplified function, modeling path loss for outdoor to indoor and pedestrian test environment, shown in [6].

We first consider a user distribution shown in figure 1(a). The results in Table 2 indicate that, both OS and CS exhibit much better performance than FS (more than $40 \%$ power savings). Compared with OS, the CS solution gives limited degradation (In both 5-sector and 6-sector cases, less than $1 \%)$. The sector partitioning results for OS and CS are also shown in Fig. 1. The sector boundaries are generally crossing

Table 2

Total transmission power. [Watt].

\begin{tabular}{lcc}
\hline $\begin{array}{l}\text { Partition } \\
\text { scheme }\end{array}$ & \multicolumn{2}{c}{ Total transmission power (Watt) } \\
\cline { 2 - 3 } & 5-sector & 6-sector \\
\hline $\operatorname{TTP}(\mathrm{OS})$ & 4.97 & 4.42 \\
$\operatorname{TTP}(\mathrm{CS})$ & 4.98 & 4.45 \\
$\quad \delta=2 \pi / 360$ & & \\
$\quad \delta=2 \pi / 36$ & 5.02 & 4.46 \\
$\operatorname{TTP}(\mathrm{FS})$ & 27.9 & 6.41 \\
\hline
\end{tabular}

low-density regions in the CS solution, while in OS, they may pass through two users very close to each other. In addition, generally speaking, when larger $\delta$ is chosen, the boundaries more likely cross two users far away. Furthermore, the computational complexity of CS is greatly reduced compared to OS. When we set the angular value for clustering to be $\delta=$ $2 \pi / 360$, the 36 users are partitioned into 19 clusters; when $\delta$ $=2 \pi / 36$, only 12 clusters left (This is obvious as the larger the interval, the more users are likely to be placed into one cluster, resulting in less clusters). Thus, approximately only $(19 / 36)^{3}$ (i.e. $14.7 \%$ as the complexity is about $\mathrm{O}\left(N M^{3}\right)$ ) computing effort is required for $\delta=2 \pi / 360$ case and $(12 / 36)^{3}$ (i.e. $3.7 \%$ ) for $\delta=2 \pi / 36$ compared to OS solution (The computing for angular-based partitioning has not been included, as it is much lower than the effort on final cluster-based sectoring). In this case, higher angular distance for clustering $(\delta)$ help to save great amount of computation effort while the boundary locations are reasonably better with the cost of little performance degradation. However, this is not always the case. For "hot spots" case, too large $\delta$ will result in very large clusters for further angular-based partitioning (In the worst case this might result in only one cluster including all users). This may greatly degrade the system performance and lose the advantage of clustering to partition through those users relatively far away. Parameters should be carefully chosen and adjusted.

A higher density user distribution is illustrated in figure 2. Here, 180 users are partitioned into 15 sectors. An extremely "hot spot" (roughly in the area of the ellipse) exists in this example, where 32 users are placed into one cluster after the coarse clustering, thus exceeding the capacity constraint of a sector. We apply the angular-based partitioning algorithm on the large cluster to divide it into some sub-clusters, with the objective of the local MinTTP (in this example, 3 sub-clusters for this large cluster, shown in figure 2(a)). In Table 3, we also show the MinTTP result for different partitioning algorithm for the 15-sector case and 18-sector case. The MinTTP of CS is still quite close to the OS solution (less than $5 \%$ in both 15 -sector and 18-sector cases). Under this user-distribution,

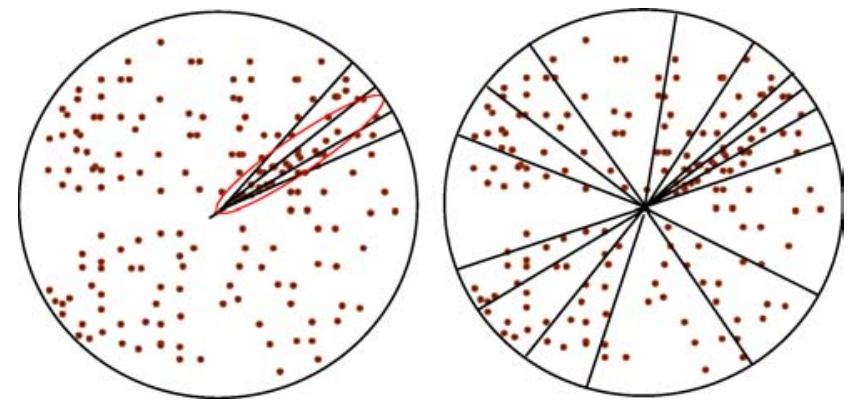

Figure 2180 -user, 15 -sector, $\delta=1$. (a). User distribution. The ellipse roughly includes one high-density cluster after coarse clustering, which is further partitioned into 3 sub-clusters with users between boundaries. (b) Sectoring result. The lines represent the final boundaries. 
Table 3

Total transmission power [Watt].

\begin{tabular}{lll}
\hline & \multicolumn{2}{l}{ Total transmission power $($ Watt $)$} \\
\cline { 2 - 3 } Partition scheme & 15 -sector & 18 -Sector \\
\hline TTP(OS) & 44.6 & 31.7 \\
TTP(CS) & 46.7 & 33.2 \\
TTP(FS) & NF & NF \\
\hline
\end{tabular}

Table 4

Comparison for different $\delta$.

\begin{tabular}{lll}
\hline $\begin{array}{l}\text { CS(180-user, } \\
18 \text {-sector) }\end{array}$ & $\begin{array}{l}\text { \# of clusters } \\
((\# / 180) 3)\end{array}$ & $\begin{array}{l}\text { TTP(Watt) } \\
\text { (degradation) }\end{array}$ \\
\hline$\delta=2 \pi / 360$ & $98(16 \%)$ & $33.2(4.7 \%)$ \\
$\delta=2 \pi / 180$ & $67(5 \%)$ & $34.6(9.1 \%)$ \\
$\delta=2 \pi / 90$ & $29(<1 \%)$ & $34.9(10 \%)$ \\
\hline
\end{tabular}

FS is not feasible because some sector(s) for FS partitioning exceed(s) the capacity constraint.

Now we investigate the result with variable $\delta$ setting. As explained earlier, with the increasing of $\delta$, the number of clusters will decrease, resulting in much faster computation. This is shown in the table as the percentage of the computing effort for CS over OS. At the same time, the performance degradation percentages are also calculated. In this case, the directivity property can't be well maintained as most of clusters require further partitioning with angular-based sectoring.

To investigate the impact from number of available sectors $(N)$, we vary $N$ from 15 to 23 for the above 180 user configuration (figure 2), where $\delta=2 \pi / 360$. The corresponding MinTTP results for OS and CS are shown in figure 3. From the experiment, we observe that, similar to OS scheme, the MinTTP results for CS algorithm also decrease with the increase of sectoring number. In general, the gap between OS and CS solutions is less than $10 \%$, and when a large number of sectors are used, the

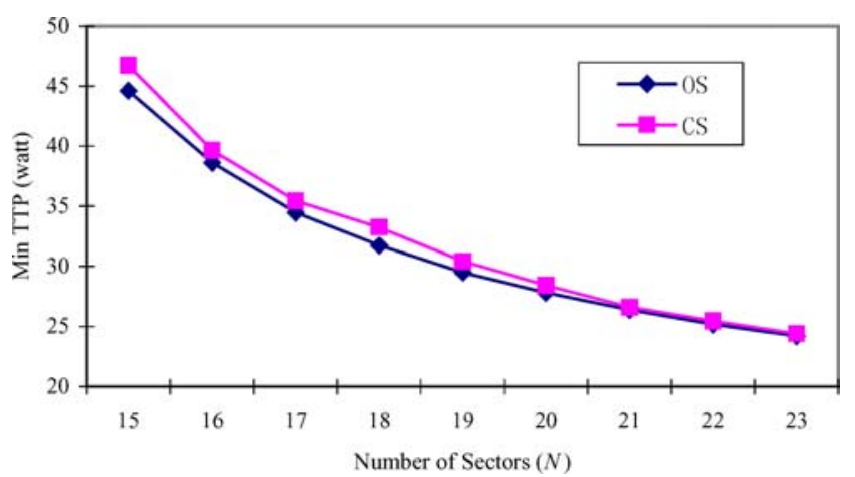

Figure 3 Minimum total transmission power for different number of sectors $k=1800$.
MinTTP achieved by CS is nearly optimal. Furthermore, as shown in the figure, the power saving trend slows down with the growth of $N$, suggesting careful adjustment on tradeoffs between the antenna cost and performance gain.

\section{Conclusion}

In this paper, we have studied the optimization problem of adaptive cell sectoring to minimize the total transmission power (TTP), while at the same time satisfying the QoS (i.e. lower bound SIR requirements) in DS-CDMA systems. As discussed in the paper, the complexity of optimal solutions is $\mathrm{O}\left(N M^{3}\right)$, and thus suffers long computation time in the highdensity cells. With the consideration to reduce system complexity and also the observation that it is better for the sector boundaries across low-density regions, we propose a clusterbased sectoring algorithm. Later section on complexity analysis shows that our cluster-based algorithm may achieve great computation gain. Specifically for high-density case, the complexity is bounded by the selected angular parameters and independent of the number of users in a cell. Furthermore, the proposed algorithms can be easily extended to accommodate multi-rate services and adjusted for more realistic systems.

Numerical results show that our cluster-based sectoring algorithm achieves comparable performance with the optimal sectoring algorithm; while at the same time, it achieves prominent savings on the computational efforts in terms of generally much lower complexity than that of the optimal algorithm. From the sectoring result, we could also find that the boundaries for our cluster-based sectoring algorithm are generally across those neighboring users relatively far away, which is beneficial from a practical point of view.

\section{Appendix A. Recurrence relation (5) is correct}

Proof: The equation can be proved by induction.

1. $n=1,1 \leq k<\frac{G}{\alpha \gamma}+1$;

The $k$ users contribute only for one sector, then $\varphi(k, n)=$ $\varphi(k, 1)=\frac{\eta \gamma}{G-(k-1) \alpha \gamma} \sum_{j \in \chi_{i}} \frac{1}{h_{j}}=\frac{\eta \gamma}{G-(k-1) \alpha \gamma} \sum_{j=1}^{k} \frac{1}{h_{j}}$.

2. $n>1, n \leq k<\frac{G}{\alpha \gamma}+i$;

Let $\varphi(i-1, n-1)$ be the optimal solution with $i-1$ consecutive users split into $n-1$ sectors. Assume the partitioning point for the last sector $\mathrm{n}$ is set to be $i$. The optimal solution $\varphi(k, n)$ takes place if and only if the solution to previous $i-1$ users is optimal with $n$-1 sectors, i.e. $\varphi(i-1, n-1)$ [10]. Then for this case, the MinTTP is $\varphi(i-1, n-1)+\frac{\eta \gamma}{G-(k-i) \alpha \gamma} \sum_{j \in \chi(i)} \frac{1}{h_{j}}$, (the last item is the TTP for $n$-th sector). Consider all the feasible placement of the partition point $i$ for 
the $n$-th sector and extract the minimum solution, i.e. $\min _{n-1<i \leq k}\left\{\varphi(i-1, n-1)+\frac{\eta \gamma}{G-(k-i) \alpha \gamma} \sum_{j \in \chi(i)} \frac{1}{h_{j}}\right\}$, here assume at least one user in a sector. This is the optimal solution for $\varphi(k, n)$.

3. $n<1, k<n, k>\frac{G}{\alpha \gamma}+i$ all are non-feasible settings and thus $\varphi(k, n)$ is set to $+\infty$.

\section{Appendix B. A cluster derived from Section 4.1 can be regarded as one particular integrated user to be used in the (6) A.}

Proof According to Section 3, the total transmission power for the system can be written as $\sum_{i=1}^{N} \frac{\eta \gamma}{G-\left(K_{i}-1\right) \alpha \gamma} \sum_{j \in \chi_{i}} \frac{1}{h_{j}}$, where $K_{i}$ is the number of users in sector $i$. As mentioned above, the users in one cluster should be put into the same sector. Assume cluster $j$ with $k$ users $C_{j}=\left\{U_{1}, U_{2}, \ldots U_{k}\right\}$ is placed into sector $i$, then the TTP contributed by $C_{j}$ is $\frac{\eta \gamma}{G-\left(K_{i}-1\right) \alpha \gamma} \sum_{s=1}^{k} \frac{1}{h_{U_{s}}}$. Thus, $C_{j}$ can be viewed as a super-user to have number factor as $k$ to contribute in the $K_{i}$ place and path loss $\frac{1}{h_{C_{j}}}=\sum_{k \in C_{j}} \frac{1}{h_{k}}$, which results in equation 6 .

$$
\begin{aligned}
\leq & \sum_{s=1}^{i-1} \frac{\eta \gamma}{G-\left(K_{s}-1\right) \alpha \gamma} \sum_{j \in \chi_{s}} \frac{1}{h_{j}}+\sum_{s=i+1}^{N-1} \frac{\eta \gamma}{G-\left(K_{s}-1\right) \alpha \gamma} \sum_{j \in \chi_{s}} \frac{1}{h_{j}} \\
& +\frac{\eta \gamma}{G-(k-1) \alpha \gamma} \sum_{j \in S_{i 1}} \frac{1}{h_{j}}+\frac{\eta \gamma}{G-(k-t) \alpha \gamma} \sum_{j \in S_{i_{2}}} \frac{1}{h_{j}} \\
= & \sum_{s=1}^{i-1} \frac{\eta \gamma}{G-\left(K_{s}-1\right) \alpha \gamma} \sum_{j \in \chi_{s}} \frac{1}{h_{j}}+\sum_{s=i+1}^{N-1} \frac{\eta \gamma}{G-\left(K_{s}-1\right) \alpha \gamma} \sum_{j \in \chi_{s}} \frac{1}{h_{j}} \\
& +\frac{\eta \gamma}{G-(k-1) \alpha \gamma} \sum_{j \in S_{i}} \frac{1}{h_{j}}
\end{aligned}
$$$$
=\text { MinTTPforN }-1 \text { cell sec toring. }
$$

\section{Acknowledgments}

This research was supported in part by grants from RGC under contracts HKUST 6402/03E and HKUST 6104/04E, a grant from NSFC under the contract 60429202, a NSFC/RGC joint grant under contract N_HKUST 605/02, and a grant from Microsoft Research under the contract MCCL02.03.EG01. J.Liu's work was supported in part by a Canadian NSERC Discover Grant.

\section{References}

[1] A. Ahmad, A CDMA network architecture using optimized sectoring, IEEE Transactions on Vehicular Technology 51(3) (May 2002) 404410.

[2] G.K. Chan, Effects of sectorization on the spectrum efficiency of cellular radio systems, IEEE Transactions on Vehicular Technology 41(3) (August 1992) 217-225.

[3] E. Dahlman, B. Gudmundson, M. Nilsson and J. Skold, UMTS/IMT2000 based on wideband CDMA, IEEE Communications Magazine 36(9) (September 1998) 70-80.
[4] K.S. Gilhousen, I.M. Jacobs, R. Padovani, A.J. Viterbi, L.A. Weaver Jr. and C.E. Wheatly, On the capacity of a cellular CDMA system, IEEE Transactions on Vehicular Technology 40 (May 1991) 303-312.

[5] R. Giuliano, F. Mazzenga and F. Vatalaro, Adaptive cell sectorization for UMTS third generation CDMA systems, Vehicular Technology Conference 1 (Spring 2001) 219-223.

[6] Guidelines for evaluation of radio transmission technologies for IMT2000, Recommendation ITU-R M.1225 (1997) 45-46.

[7] C.-L. I and R.D. Gitlin, Multi-code CDMA wireless personal communications networks, in: IEEE Proc. ICC'95 (1995), pp. 10601064.

[8] K. Kim, Handbook of CDMA System Design Engineering, and Optimization, in: Prentice Hall PTR, 2000.

[9] B. Li, X. Deng, M.J. Golin and K. Sohraby, On the optimal placement of web proxies in the Internet: Linear topology in: $\{\backslash$ it the 8th IFIP Conference on High Performance Networking (HPN'98).

[10] Z. Liu, M.J. Karo, M.E. Zarki and K.Y. Eng, A demand-assignment access control for multi-code DS-CDMA wireless packet (ATM) networks, in: IEEE Proc. INFOCOM'96 (1996) pp. 713-721.

[11] A.F. Naguib, A. Paulraj and T. Kailath, Capacity improvement with base-station antenna arrays in cellular CDMA, IEEE Transactions on Vehicular Technology 43(3) (August 1994) 691-698.

[12] T.S. Rappaport, $\{\backslash$ it Wireless Communications: Principles $\} \&$ Practice, (Prentice Hall, 1996).

[13] C.U. Saraydar and A. Yener, Adaptive cell sectorization for CDMA systems, IEEE Journal on Selected Areas in Communications 19(6) (June 2001) 1041-1051.

[14] P. Viswanath, D.N.C. Tse and R. Laroia, Opportunistic beamforming using dumb antennas, IEEE Transactions on Information Theory 48(6) (June 2002) 1277-1294.

[15] J.S. Wu, J.K. Chung and C.C. Wen, Hot-spot traffic relief with a tilted antenna in CDMA cellular networks, IEEE Transactions on Vehicular Technology 47(1) (February 1998) 1-9.

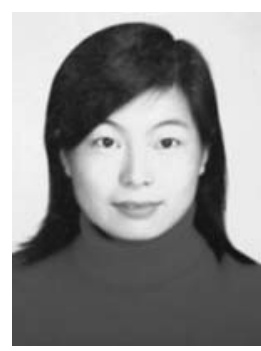

Jihui Zhang received her B.S. degree from Computer Science Department at Fudan University, China. She is working toward the Ph.D. degree in the Computer Science Department at Hong Kong University of Science and Technology. Her research interests include the resource management in CDMA cellular networks and wireless ad-hoc networks.

E-mail: jhzhang@cs.ust.hk

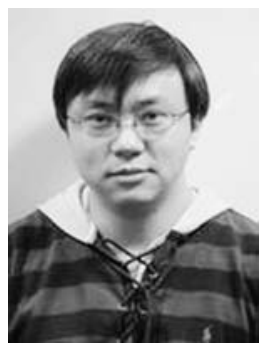

Bo Li received his B. Eng. (summa cum laude) and M. Eng. degrees in the Computer Science from Tsinghua University, Beijing in 1987 and 1989, respectively, and the Ph.D. degree in the Electrical and Computer Engineering from University of Massachusetts at Amherst in 1993. Between 1993 and 1996, he worked on high performance routers and ATM switches in IBM Networking System Division, Research Triangle Park, North Carolina. Since 1996, he has been with the Department of Computer Science, Hong Kong University of Science and Technology. He has held an adjunct researcher position at the Microsoft Research Asia (MSRA), Beijing, China. His research interests are on adaptive video multicast, packet scheduling and dynamic routing in optical networks, resource management in mobile wireless systems, scheduling and energy efficient routing in ad hoc networks, across layer design for sensor networks, and content distribution and replication. He has published 70 some journal papers and held several patents in above areas.

He received the Outstanding Oversea Young Scientist Award from National Natural Science Foundation of China in 2004. He has been on editorial board 
for 16 journals and involved in organizing over 40 conferences, esp. IEEE Infocom since 1996. He was the Co-TPC Chair for IEEE Infocom 2004.

E-mail: bli@cs.ust.hk

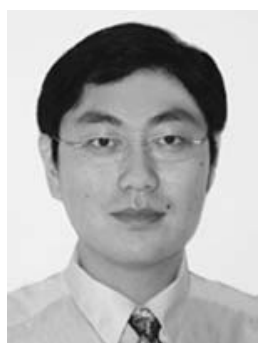

Jiangchuan Liu received the B.Eng degree (cum laude) from Tsinghua University, Beijing, China, in 1999, and the Ph.D. degree from The Hong Kong University of Science and Technology in 2003, both in computer science.

He is currently an assistant professor in the School of Computing Science, Simon Fraser University,

$\mathrm{BC}$, Canada, and was an assistant professor at The Chinese University of Hong Kong from 2003 to 2004. He was a recipient of Microsoft research fellowship (2000), a recipient of Hong Kong Young Scientist Award (2003), and a co-inventor of one European patent (granted) and two US patents (pending). He won first-class honors in several regional and national programming contests.

His research interests include Internet architecture and protocols, media streaming, wireless ad hoc networks, and service overlay networks. He serves as TPC member for various networking conferences, including IEEE INFOCOM'04 and '05. He was TPC Co-Chair for The First IEEE International Workshop on Multimedia Systems and Networking (WMSN'05), Information System Co-Chair for IEEE INFOCOM'04, and a guest-editor for ACM/Kluwer Journal of Mobile Networks and Applications (MONET), Special Issue on Energy Constraints and Lifetime Performance in Wireless Sensor Networks. He is a member of IEEE and IEEE Communications Society, and an elected member of Sigma Xi.

E-mail: jcliu@cs.sfu.ca 